\title{
Treatment of Feline Dermatophytosis with an Inactivated Fungal Vaccine
}

\author{
D.K. Westhoff ${ }^{*}, 1,3$, M.-C. Kloes ${ }^{1}$, F.X. Orveillon ${ }^{1}$, D. Farnow ${ }^{1}$, K. Elbers ${ }^{1}$ and R.S. Mueller ${ }^{2}$ \\ ${ }^{1}$ Boehringer Ingelheim Vetmedica GmbH, Ingelheim, Germany \\ ${ }^{2}$ Medizinische Kleintierklinik, Ludwig Maximilian University, Munich, Germany \\ ${ }^{3}$ RRD International LLC, Rockville MD, USA
}

\begin{abstract}
The efficacy of an inactivated vaccine for the treatment of feline dermatophytosis was investigated in a placebo-controlled-double-blind multi-centre GCP study in Europe. Fifty-five client-owned cats with dermatophytosis caused by Trichophyton mentagrophytes or Microsporum canis, confirmed by fungal culture, were treated with either three intramuscular injections of vaccine or placebo. Treatment was applied as three intramuscular injections of vaccine or placebo every other week. Clinical symptoms were assessed at inclusion, day 14, 28 and 42. The number of lesions was counted and severity was judged based on a scoring system. Efficacy was evaluated for the reduction of the number of lesions as well as for a combined assessment of lesion severity $\mathrm{x}$ number of lesions. The primary endpoint was not met for the total population of cats, but was met for cats $<1$ year of age and for cats with a first infection. The vaccine was significantly better than placebo in cats with a first infection (sum of lesions: $p=0.0446$; summed score $x$ number: $\mathrm{p}=0.0405$ ) and in young cats (sum of lesions: $\mathrm{p}=0.0424$; summed score $\mathrm{x}$ number: $\mathrm{p}=0.0304$; mean score $\mathrm{x}$ number: $\mathrm{p}=0.0177$ ). In cats with higher numbers of more severe lesions, the difference between the two treatment groups was more apparent. Severely affected exotic cats also showed an improvement using these parameters. Based on this study, the investigated inactivated vaccine may be considered as part of a treatment protocol to accelerate healing from clinical signs of dermatophytosis in severely affected cats, in young cats and those with a first infection.
\end{abstract}

Keywords: Feline dermatophytosis, Microsporum, Trichophyton, therapeutic vaccination, fungal infection.

\section{INTRODUCTION}

Feline dermatophytosis is an infection of the superficial keratinised tissues of the cat by dermatophytes. The most common cause of dermatophytosis in the cat is the fungus Microsporum canis [1-5]. Two other species, Microsporum gypseum and Trichophyton mentagrophytes can also cause dermatophytosis in the cat, but with a lower incidence [2-5]. A similar distribution pattern of these dermatophytes in cats has been reported for European and American countries [2, 3, 6-10]. Dermatophytosis caused by $M$. canis frequently affects young cats and is more often observed in exotic and long-haired cats (e.g. Persians, Angora), than in domestic short-haired cats $[3-5,8,9]$. An infection with a fungal pathogen is dependent on the current health status of the cat, stress factors, number of spores, hygienic status and possibly genetic predisposition. Cats under immunosuppressive treatment are at a higher risk for developing an infection [5, 9, 11]. Dermatophytosis is highly contagious for other animals in the household as well as for family members and decontamination of the environment is highly recommended $[5,12,13]$.

The currently recommended therapies are often combinations of systemic and topical treatments supported by the partially or entirely clipping of the cats and are associated with varying levels of owner compliance $[4,5]$. The duration

*Address correspondence to this author at the 19112 Munger Farm Road, Poolesville MD, 20837, USA; Tel: 001-301-349-5067; Fax: 001-301-7626154; E-mail: dwsthff@verizon.net, dwesthoff@ rrdintl.com of treatment varies and is depending on the housing conditions and the type of treatment or combination of treatments $[4,5,9]$. Griseofulvin and azoles such as ketoconazole and itraconazole are reported as systemic treatments $[4,5$, 14, 15]. The typical topical treatments used for rinsing, dipping or shampooing are chlorhexidine solution, enilconazole or miconazole $[4,9,16]$. The use of antifungal vaccines as a therapeutic option for humans and animals where the infective strains are resistant against other treatments has been investigated since 1944 [17-19]. Vaccines as prophylactic or therapeutic treatment against dermatophytosis have been developed for cattle, horses, foxes, guinea pigs, cats and dogs [20-26]. Several attempts have been made to develop fungal vaccines for prevention and/or therapy of dermatophytosis in cats, such as laboratory prepared fungal cell wall vaccines [27, 28], an inactivated broad-spectrum dermatophyte vaccine [29] or a liveattenuated dermatophyte vaccine [30]. None of the investigated vaccines for cats showed sufficient protection against challenge exposure $[4,21,27,28,31]$. A vaccine for prophylaxis of $M$. canis infection in cats and dogs is approved in Germany (Rivac Mikroderm, Riemser Arzneimittel AG, Germany). Another vaccine (Insol ${ }^{\circledR}$ Dermatophyton, Boehringer Ingelheim, Germany) is licensed for the therapeutic and prophylactic use in horses, cats and dogs in several European countries [32, 33].

The aim of this placebo-controlled multi-centre field study in Europe was to investigate a pentavalent vaccine for the therapeutic treatment of clinical symptoms of dermatophytosis in cats caused by $M$. canis, M. gypseum and/or T. mentagrophytes. 


\section{MATERIALS AND METHODOLOGY}

The study was conducted as a double-blind, randomized, placebo-controlled multi-centre clinical field study enrolling cats at fifteen (15) sites in Denmark, France and Germany from June 2004 to July 2007.

\section{Study Design}

Cats were distributed to treatment group 1 or 2 by a randomization protocol prepared by a statistician. Group 1 received three treatments with the therapeutic vaccine and group 2 received three treatments with a placebo. Treatments were administered at 14-day intervals on day 0 , day 14 and day 28. Lesions were assessed on day 0, day 14, day 28 and day 42. Skin/hair coat samples were collected on day 0 and day 42. Cats in this efficacy evaluation were part of a crossover study to also evaluate the safety of the vaccine. Cats in the placebo group received treatment with the vaccine following the final efficacy assessment. The investigators, the sponsor and the cat owners were blinded until data-lock and submission of the complete dataset to the statistician.

\section{Study Participants}

Fifty-five (55) client-owned cats, 26 female and 29 male cats, were included in total, presenting several different breeds, including domestic short-haired cats, domestic longhaired cats, Persian, Main Coon, Holy Birman, Norwegian Forest, Colour Point, Selkirk Rex, Russian Blue Mix and Angora. Cats not belonging to the domestic short-haired breed were later compiled into a group named "exotic cats" for simplification. Cats had to be healthy (except for clinical signs of skin disease), older than twelve weeks of age and currently not being treated with immunosuppressive drugs. Dermatophytosis was clinically diagnosed by the investigator and confirmed by a laboratory fungal culture, including classification of genera and species of the causative fungal pathogen. If a different antimycotic treatment had already been administered, a wash-out period of four weeks before administration of the first study treatment was required. No cats from multiple cat households, shelters or catteries were included. In two instances, two cats from the same household were affected and in these cases, both cats from each household were included in the study. Cat owners were provided with a disinfectant and requested to wash/disinfect bedding, toys and grooming material of the affected cat once weekly to help prevent re-infection.

\section{Examination of Clinical Signs}

At day $0,14,28$ and 42, the lesions of the skin and hair coat at different body locations (face, ears, back, chest, abdomen, front leg, hind leg, tail, claws) were examined. The number of lesions was recorded for each body location. The investigators were trained to evaluate the severity of lesions at the different body locations using a scoring system (Table 1) and the highest score per body location was recorded.

\section{Exclusions from the Study Evaluation}

Four cats were excluded due to non-compliance of the owners and one cat died due to an event not related to the
Table 1. Scoring System for the Judgment of Lesion Severity of the Dermatophytosis

\begin{tabular}{|c|l|}
\hline Score & \multicolumn{1}{|c|}{ Clinical Signs } \\
\hline \hline 1 & Skin without abnormal findings \\
\hline 2 & $\begin{array}{l}\text { Hairless areas/areas of thinning hair with } \\
\text { local inflammation (erythema and swelling) }\end{array}$ \\
\hline 3 & Hairless areas with mild crusts/scales/exudate \\
\hline 4 & Hairless areas with prominent crusts/scales/exudate \\
\hline 5 & Inflamed plaques, pustules and papules \\
\hline
\end{tabular}

study treatment (cardiomyopathy, polycystic kidney disease). Thus efficacy on the reduction of lesions was evaluated for a total number of 50 cats. Hair/fur samples for day 42 were not assessed for four cats. Therefore the fungal culture results at day 42 are only available for 46 cats (Table. 2).

Table 2. Number of Study Cats for the Statistical Evaluations and Distribution to the Treatment Groups

\begin{tabular}{|c|c|c|c|c|c|}
\hline \multirow{2}{*}{$\begin{array}{l}\text { Treatment } \\
\text { group }\end{array}$} & \multicolumn{2}{|c|}{$\begin{array}{l}\text { Number of Cats with } \\
\text { at least One Lesion }\end{array}$} & \multicolumn{3}{|c|}{$\begin{array}{c}\text { Number of Cats with at least } \\
\text { One Lesion score }>2 *\end{array}$} \\
\hline & $\begin{array}{c}\text { Score } \\
>1 *\end{array}$ & $\begin{array}{c}\text { Score } \\
>2 *\end{array}$ & $\begin{array}{l}\text { Young } \\
\text { cats }\end{array}$ & $\begin{array}{c}\text { First } \\
\text { infection }\end{array}$ & $\begin{array}{l}\text { Exotic } \\
\text { cats }\end{array}$ \\
\hline Vaccine & 26 & 17 & 9 & 13 & 7 \\
\hline Placebo & 24 & 18 & 10 & 15 & 7 \\
\hline Total & 50 & 35 & 19 & 28 & 14 \\
\hline
\end{tabular}

\section{Study Treatment and Route of Administration}

The vaccine was formulated as an aqueous solution containing $10 \times 10^{6}$ to $12 \times 10^{6}$ microconidia of each of the following strains: T. mentagrophytes (strain no. 1032), $M$. canis (strain no. 1393), M. canis var. distortum (strain no. 120), M. canis var. obesum (strain no. 1311), M. gypseum (strain no. 59) and $0.04 \mathrm{mg}$ thiomersal per $1 \mathrm{ml}$. From each of the fungal strains $4.0 \times 10^{6}$ microconidia were used for cultivation. Microconidia were counted using a Buerker counting chamber. Cultivation of the fungal strains was done separately for each strain under sterile conditions in modified malt agar for 15 to 20 days at $26-28^{\circ} \mathrm{C}$. After harvesting, each fungal mass was homogenized with an Ultra-turrax at $10,000 \mathrm{rpm}$. Following a homogenization step, each individual fungal strain suspension was adjusted to a microconidia count of $55-65 \times 10^{6} / \mathrm{ml}$. The microconidia suspension was allowed to germinate. The culture with the germinated microconidia was inactivated by adding sterile thiomersal solution to a final concentration of $40 \mu \mathrm{g} / \mathrm{ml}$ microconidia fungal suspension before a final filtration process. This final filtration process leads to a concentration of the microconidia in the final suspension. After filtration, the suspensions containing the five inactivated fungal strains were combined at equal quantities and stored at $2-8{ }^{\circ} \mathrm{C}$. 
A placebo, not containing the active ingredients was used to mimic the turbidity of the investigational vaccine (aqueous solution: $0.04 \mathrm{mg} / \mathrm{ml}$ thiomersal and Intralipid ${ }^{\circledR} 10$ (Baxter Medication Germany GmbH, Germany) at OD 1.8. The investigators received pre-packed sets of vials per cat (one vial per injection), labeled with a specific case number and containing either vaccine or placebo according to the randomization protocol. Vaccine or placebo were administered three times on the scheduled days $(0,14$ and 28) as deep intramuscular injection into the muscles of the hind legs of the cats, alternating between right and left legs.

\section{Fungal Cultures}

Specimens from one to three different affected parts of the skin or hair coat were collected on day 0 and day 42 . The margins of the affected areas were disinfected with $70 \%$ alcohol, to avoid contamination with other pathogens. The alcohol was allowed to evaporate before sampling. Skin scrapings were taken with a sterile scalpel and hair specimens were collected with a sterile tooth brush combed over the periphery of the affected skin. Each sample was divided into two aliquots and two different commercially available test kits were inoculated with the aliquots (Mycodetect, WDT Germany; InTray ${ }^{\mathrm{TM}}$ DM, Janssen, Germany). The test kits were incubated at room temperature (approximately $22^{\circ} \mathrm{C}$ ) at a central laboratory (Serumwerk Memsen, WDT, Germany) based on their standard operating procedures. The presence of dermatophytes was indicated by a colour change turning the agar to red. A visible, positive test result was expected within three to seven days. The culture media with the inoculated material were incubated aerobically at $25{ }^{\circ} \mathrm{C}$ for approximately five weeks. Suspected colonies were sub-cultivated and incubated on Sabouraud-Agar at $25^{\circ} \mathrm{C}$. Primary cultures were considered negative at the earliest after five weeks. Sub cultures of colonies from primary cultures were examined a least once weekly (macroscopically and microscopically) with respect to accepted culture and morphological criteria (e.g. growth rate, shape and colour of colonies, presence, number and morphology of micro- and microconidia).

\section{Statistical Evaluation}

The primary endpoint was the reduction of lesions at day 42. This was assessed by evaluating the reduction in the number of lesions and lesion score severity $\mathrm{x}$ number of lesions (SAS, release 8.02) with the individual cat as experimental unit. Data were cumulated over all affected locations per animal (sum) and mean values were calculated per animal as the sum divided by the number of affected locations. The difference per cat between day 0 and day 42 was calculated and the treatment groups were compared with respect to differences using Wilcoxon Mann-Whitney tests with a statistical significance level of $\mathrm{p}=0.05$. All parameters were calculated for the study days $0,14,28$ and 42. The recovery between study days 0 and 14, 0 and 28, and between 0 and 42 was also calculated per animal as change from baseline 'Day' X - 'Day' 0 and appear as negative values. The statistical evaluation showed that the data were not normally distributed. For this reason, mean values were not valid and median values were used.

\section{RESULTS}

\section{Evaluation of Clinical Signs}

Lesions were seen most frequently on the face, the ears and the back, followed by legs and abdomen. Lesions at the tail and the claws were seen less often. The distribution of the number of lesions was similar in both treatment groups. Lesion severity (please refer to Table $\mathbf{1}$ for details) at day 0 was more often scored with a 2 or 3 than with a 4 or 5 . Lesions severity scores of $1-2$ were regarded as minor and scores 3 - 5 were regarded as more severe. Severity score 5 was given only three times at day 0 in the vaccine group $(2 \mathrm{x}$ face, $1 \mathrm{x}$ fore legs) and five times in the placebo group (1x face, $1 \mathrm{x}$ ears, $1 \mathrm{x}$ hind legs, $2 \mathrm{x}$ back). At day 42 no severity score 4 or 5 was given for any lesion.

Efficacy evaluations were performed for the total number of 50 cats and specifically for the group of 35 cats with severe infections (severity score $>2$ ). Sub-evaluations were performed within the group of cats with severe infections for 19 young cats $(<12.5$ months of age), 14 exotic cats (not domestic-short hair) and 28 cats infected for the first time with dermatophytes (Table 2). A sub-evaluation for female and male cats did not reveal any significant differences or trends for the healing of the dermatophytosis or in the appearance and number of lesions (data not shown).

The primary endpoint for the efficacy assessment over all cats was not met, however the median values for the reduction of lesions from day 0 until day 42 for severely affected cats (with a lesion severity score $>2$ ) show a slightly faster recovery for the vaccinated cats compared to placebo treated cats over the course of the study. The differences between the two groups were more apparent, when the number of lesions was combined with the severity of lesions but did not reach statistical significance. Table $\mathbf{3}$ shows the results for the efficacy assessment for all cats with severe clinical signs over the course of the study as a change from baseline (negative values). Table $\mathbf{3}$ shows the median and the p-values for the assessed cats based on the sum of the lesion score per single cat and the mean of all lesion scores per single cat.

Table 3. Improvement of Dermatophytosis in Cats with Severe Clinical Symptoms: Median and p-Values for the Time between Day 0 and 42 for the Study Cats Based on the Sum of the Lesion Scores per Single Cat and the Mean of all Lesion Scores per Single Cat

\begin{tabular}{|c|c|c|c|c|}
\hline \multirow{2}{*}{ Group } & \multicolumn{2}{|c|}{$\begin{array}{c}\text { Lesions [Sum (Severity } \\
\text { Score x Number)] }\end{array}$} & \multicolumn{2}{c|}{$\begin{array}{c}\text { Lesions [Mean (Severity } \\
\text { Score x Number)] }\end{array}$} \\
\cline { 2 - 4 } & Median** & p-value* & Median** & p-value* \\
\hline \hline $\begin{array}{c}\text { Vaccine } \\
(\mathrm{N}=17)\end{array}$ & -10.000 & 0.0558 & -4.500 & \\
\cline { 1 - 1 } $\begin{array}{c}\text { Placebo } \\
(\mathrm{N}=18)\end{array}$ & -2.000 & $\mathrm{~ns}$ & $\begin{array}{c}0.1091 \\
\mathrm{~ns}\end{array}$ \\
& & -2.000 & \\
\hline
\end{tabular}

* Wilcoxon Mann-Whitney test: ns: not significant: $p>0.05$;

** Median values are shown because data were not normally distributed.

The primary endpoint of the efficacy assessment was met for cats under the age of one year. Statistically significant differences between vaccine and placebo were observed for young cats with a severe dermatophytosis over the course of 


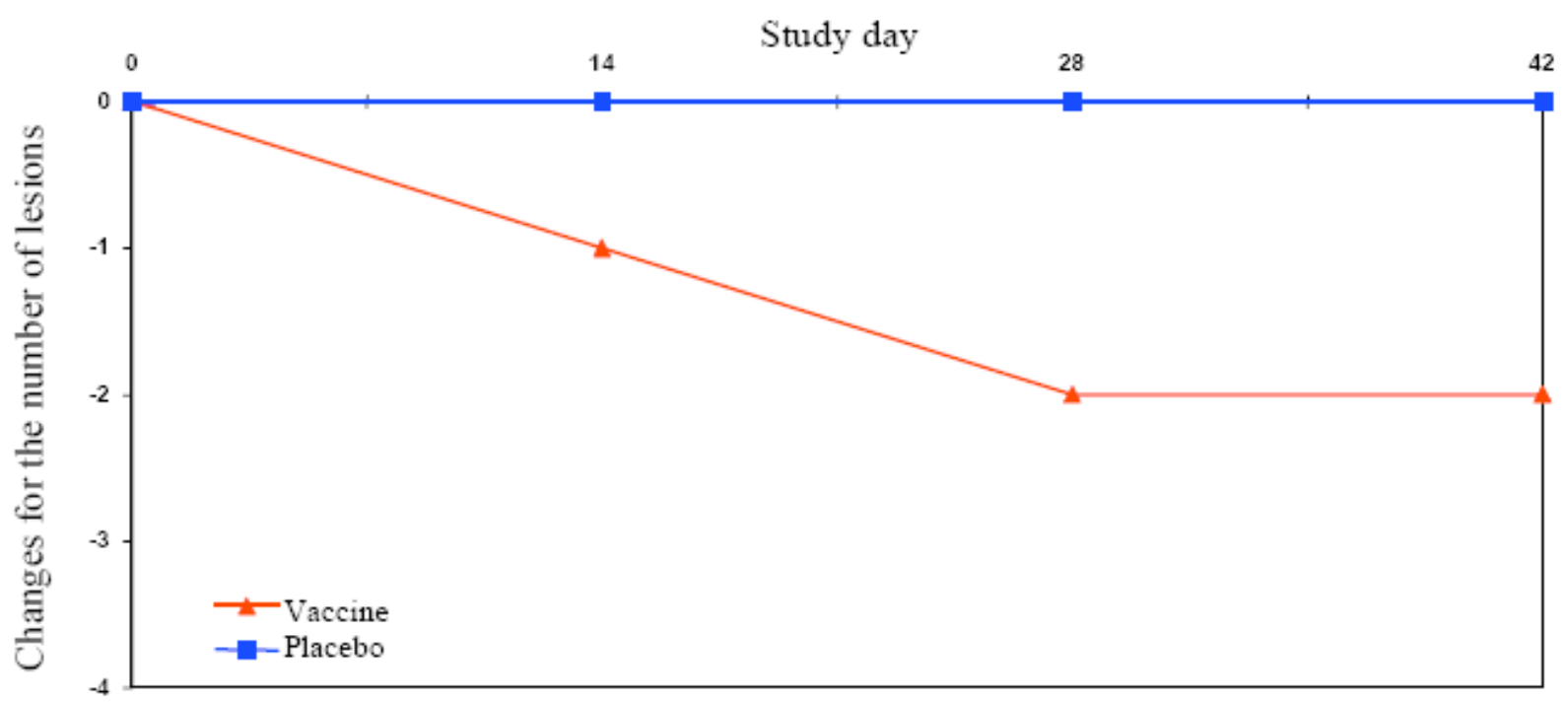

Fig. (1). Healing of lesions in severely affected cats $<12.5$ months of age at day of inclusion; comparison of vaccinated cats $(\mathrm{N}=9)$ and placebo treated cats $(\mathrm{N}=10)$ over the course of the study. Median values for the number of lesions per cat $($ day $0-42: \mathrm{p}=0.0424)$ are shown because data were not normally distributed.

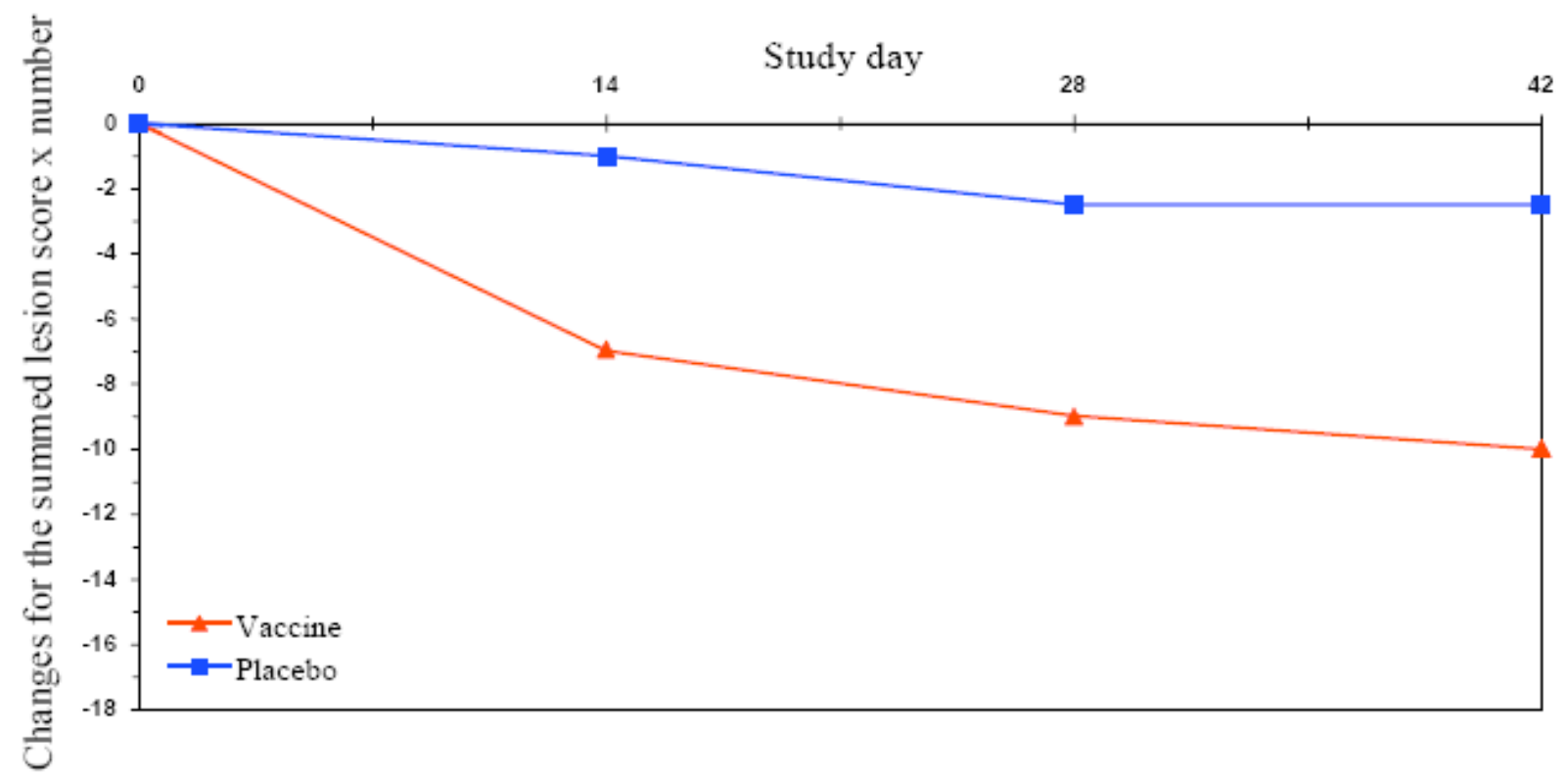

Fig. (2). Healing of lesions in severely affected cats < 12.5 months of age at day of inclusion; comparison of vaccinated cats $(\mathrm{N}=9)$ and placebo treated cats $(\mathrm{N}=10)$ over the course of the study. Median values for the summed lesion score $\mathrm{x}$ number of lesions per cat (day $0-14: \mathrm{p}=0.0177$, day $0-42: \mathrm{p}=0.0857)$ are shown because data were not normally distributed.

the study for the reduction of the number of lesions (day $0-42: p=0.0424$, Fig. 1). The median values show the improvement for the treated cats, while no improvement was observed after placebo treatment. Recovery of lesions was also assessed as combination of severity and number of lesions in young cats over the course of the study. Significant differences between the two treatment groups were seen for the summed lesion score per single cat $\mathrm{x}$ number of lesions (day $0-14: p=0.0177$, Fig. 2) and for the mean lesion score per single cat $\mathrm{x}$ number of lesions (day 0 - 14: $\mathrm{p}=0.0304$ ).

The primary endpoint of the efficacy evaluation was also met for cats with a first infection. Vaccinated cats with a first infection which exhibited severe lesions showed a faster recovery than the placebo treated cats. The development of lesions over the course of the study (day 0 to day 42) assessed as number of lesions and as the summed lesion score $\mathrm{x}$ number of lesions showed statistically significant differences $(p=0.0446$ and $p=0.0405$, respectively $)$ between the two groups (Table 4, Fig. 3).

An evaluation including only severely affected cats that were not of the domestic short-haired breed also showed a faster recovery from clinical signs for vaccinated cats compared to placebo treated cats, although this was not statistically significant (Fig. 4). 


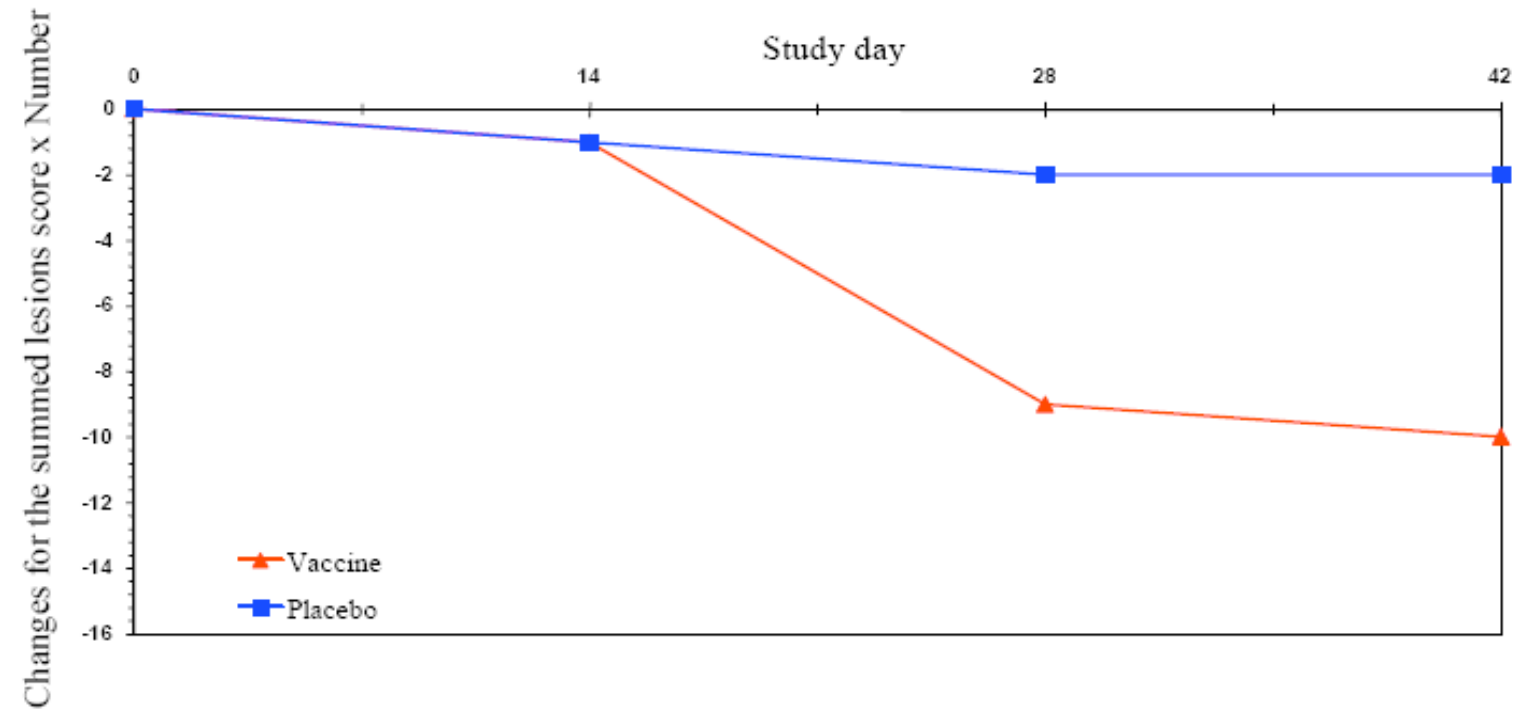

Fig. (3). Healing of lesions in severely affected cats with a first fungal infection; comparison of vaccinated cats $(\mathrm{N}=13)$ and placebo treated cats $(\mathrm{N}=15)$ over the course of the study. Median values for the summed lesion score $\mathrm{x}$ number of lesions per cat $(\mathrm{day} 0-42: \mathrm{p}=0.0405)$ are shown because data were not normally distributed.

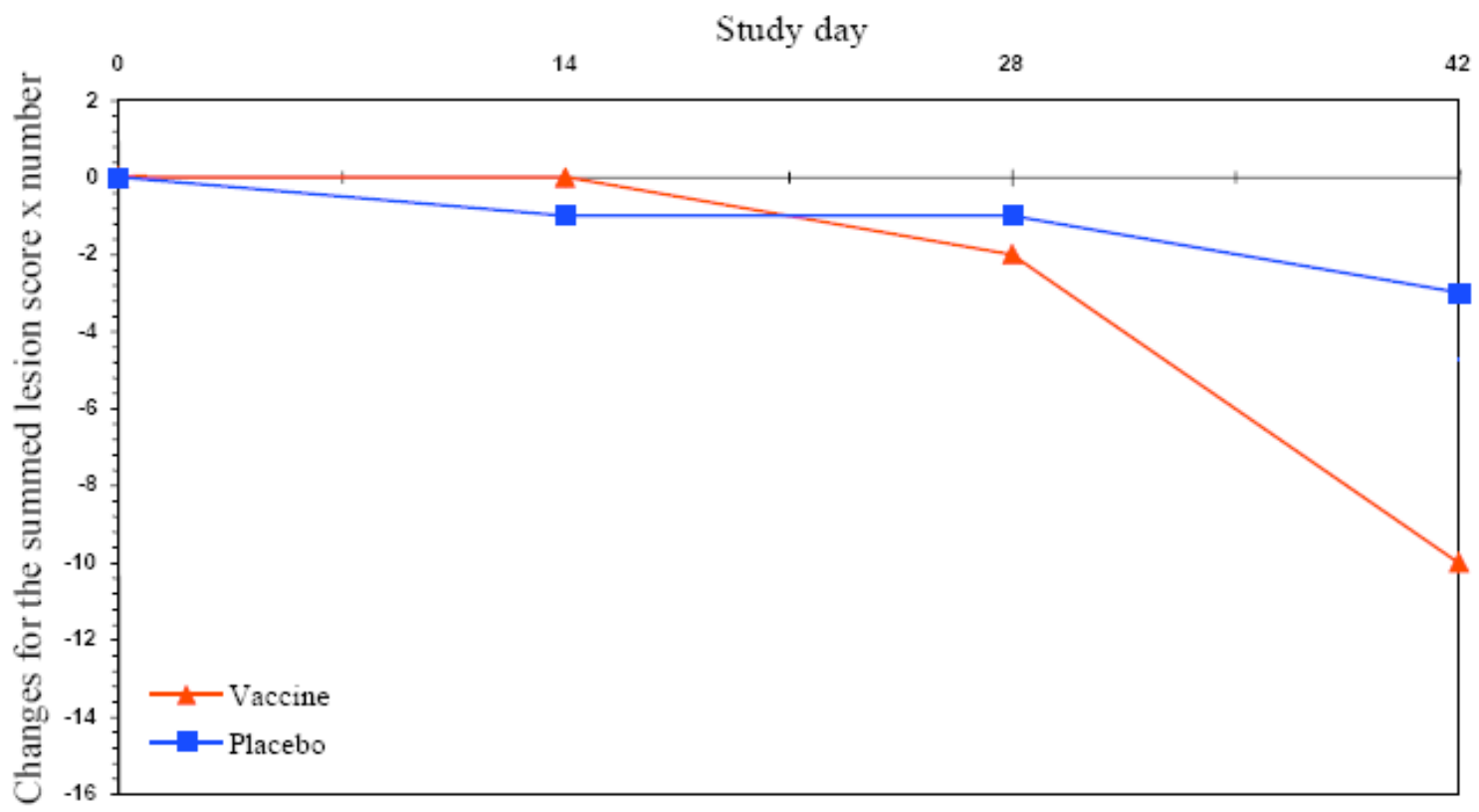

Fig. (4). Healing of lesions in severely affected exotic cats; comparison of vaccinated cats $(\mathrm{N}=7)$ and placebo treated cats $(\mathrm{N}=7)$ over the course of the study. Median values for the summed lesion score x number of lesions per cat are shown because data were not normally distributed.

Table 4. Improvement of Lesions in Severely Affected Cats with a First Infection of Dermatophytosis: N, Median and p-Value for the Time between Day 0 and day 42

\begin{tabular}{|c|c|c|c|c|}
\hline Statistical Evaluation & Group & $\mathbf{N}$ & Median* & P-value** \\
\hline \hline \multirow{2}{*}{ Number of lesions } & Vaccine & 13 & -1.000 & \multirow{2}{*}{0.0446} \\
\cline { 2 - 4 } & Placebo & 15 & 0.000 & \\
\hline \multirow{2}{*}{$\begin{array}{c}\text { Summed lesion score x } \\
\text { number }\end{array}$} & Vaccine & 13 & -10.000 & \multirow{2}{*}{0.0405} \\
\cline { 2 - 4 } & Placebo & 15 & -2.000 & \\
\hline
\end{tabular}

* Median values are shown because data were not normally distributed.

** p-value of the Wilcoxon Mann-Whitney test for comparison between group: significant $=\mathrm{p}<0.05$.

\section{Fungal Cultures}

Table 5 shows the results of the fungal cultures at day 0 and day 42. Twenty-nine (29) cats in the vaccine group and 23 cats of the placebo cats were infected with $M$. canis. At day $0, T$. mentagrophytes was identified three times in the placebo group, but not in the vaccine group. M. gypseum infection was not found in any of the study cats. At the end of the study (day 42), 18 out of 24 vaccinated cats and 12 out of 22 placebo cats still showed positive culture results. The difference between the two treatment groups was not statistically significant. 
Table 5. Results of the Laboratory Fungal Culture Test for all Study Cats at Day 0 and Day 42

\begin{tabular}{|c|c|c|c|c|}
\hline \multirow{2}{*}{ Fungal Strain } & \multicolumn{2}{|c|}{ Study Day 0 } & \multicolumn{2}{c|}{ Study Day 42 } \\
\cline { 2 - 5 } & $\begin{array}{c}\text { Vaccine } \\
\text { Group }\end{array}$ & $\begin{array}{c}\text { Placebo } \\
\text { Group }\end{array}$ & $\begin{array}{c}\text { Vaccine } \\
\text { Group }\end{array}$ & $\begin{array}{c}\text { Placebo } \\
\text { Group }\end{array}$ \\
\hline \hline M. canis & 29 & 23 & 17 & 12 \\
\hline M. gypseum & 0 & 0 & 0 & 0 \\
\hline T. mentagrophytes & 0 & 3 & 1 & 0 \\
\hline Negative & 0 & 0 & 6 & 10 \\
\hline $\begin{array}{c}\text { Total number of } \\
\text { cats }\end{array}$ & 29 & 26 & 24 & 22 \\
\hline
\end{tabular}

\section{DISCUSSION}

Feline dermatophytosis is a disease primarily caused by $M$. canis, $M$. gypseum or T. mentagrophytes [1-5]. The currently recommended treatments are often combinations of systemic and topical treatments of different duration and different intensity supported by total or partial clipping of the cats. Available treatment options are frequently not sufficient and are not satisfying to the owners due to the frequency or differences of treatment. The need for a prophylactic or therapeutic vaccine has been discussed for many years and several attempts have been made to develop such a vaccine for cats $[5,20,21,27-31]$.

In this study we compared the efficacy of a new therapeutic vaccine to a placebo in a double-blinded field study. The tested cats in this study had a confirmed dermatophytosis caused by $T$. mentagrophytes or M. canis. Infected cats were included in the study over a period of three years in three European countries. In contrast to a laboratory study with standardized conditions, the conduct of a study with client owned cats deals with multiple variables, such as different housing conditions, breeds, varying levels of owner compliance and support. However, such a study represents the conditions present in the daily clinical practice. No analysis of data based on the country location of the site was performed. All participating sites were located in Middle and Northern Europe and incidence of the fungi in cats has been reported to be similar for several European countries $[2,6,10]$.

Evaluations were focused on cats with severe dermatophytosis. Untreated cats with severe dermatophytosis have a decreased chance of a spontaneous remission compared to mild infections which maybe self-limiting and for which a spontaneous remission may be observed within a period of a few months following clinical diagnosis of the disease [1, 34]. Consequently the clinical response of cats with a severe disease is more relevant to daily practice. Some severely affected cats may even develop persistent infections and resistance to current treatments [9]. The youngest age for inclusion of cats into the study was twelve weeks of age, a common age for first vaccinations, when an immune response to vaccines can be expected in the cat [35-37]. Dermatophytosis that is caused by $M$. canis frequently affects young cats, usually less than one year of age [3-5, 810]. The specific analysis of data for cats less than one year of age at day of inclusion showed a significant improvement after treatment with this pentavalent vaccine compared to placebo. These young vaccinated cats, which were also likely naïve to fungal infections, showed a faster rate of healing of lesions than cats treated with a placebo. Cats with a first infection by dermatophytes at any ages also showed a significantly faster recovery from clinical signs following therapeutic vaccination than the placebo treated cats. These cats with a first infection ranged in age from 12.5 weeks to 10.9 years and included short-haired and exotic long-haired and short-haired cats. Thus, treating cats infected with dermatophytes with the fungal vaccine evaluated in this study may be particularly useful, if they are infected with a dermatophyte for the first time. The therapeutic vaccine tested in this study provided a statistically significant improvement in two groups of cats; the young cats, less than one year of age and those cats with first infection. These two groups of cats make up important groups, seen by veterinarians in daily practice that require treatment to limit the spread or contain the disease. In this study severely affected cats (scores $>2$ ) treated with the therapeutic vaccine have an accelerated healing of lesions when compared to placebo treated cats. In the treated cats there was a clear trend towards faster healing of lesions, although this was not significant $(p=0.056$; Table 3$)$ the vaccine provided a reduction in lesion score severity multiplied with the number of lesions.

The results from the fungal culture tests show that the vaccine does not eliminate the spores during the short time of observation in this study. A number of cats were still positive at the end of the treatment period despite a significant improvement in lesion scores or clinical remission. Continuing the study for a longer time may have resulted in a higher rate of complete elimination of the dermatophytes and continuing healing. However, most of the cats were followed until end of the safety evaluation at day 84 and for these vaccinated cats no further treatment was required. Alternatively, a combination of topical or systemic antifungal therapy and vaccination may increase the number of cats with negative culture after six weeks. However, positive culture tests after recovery from clinical signs may also be a sign of a contaminated environment and recontamination of the cat. If cats are completely recovered from clinical signs, but still show positive fungal culture test, a better disinfection and decontamination of the environment is indicated. In this study, cat owners were asked to disinfect bedding, toys and grooming material on a regular basis, but a control by the investigator was not possible. Despite the possibility that cats with no clinical signs may still be a carrier for fungal spores for a long time, it is the goal of an effective fungal treatment to eliminate all spores from the cats' fur. The importance of environmental decontamination has been stated previously, especially for $M$. canis, because of its zoonotic potential $[5,8,12,13]$. Best results will likely be achieved when therapy is supported by disinfection of the cat's toys, bedding and grooming material. Even with regular disinfection programs a severely contaminated environment can remain positive for spores for an extended period [4, 5, 13]. Due to the increase of cats kept in-door with close contact to the owner, treatment of dermatophytosis should occur as soon as possible to accelerate the healing process and to reduce the number of spores and therefore risk of 
exposure for household members, pets and owners alike. Exotic long-haired cats such as Persians or Holy Birman and short-haired cats, such as Siamese, are infected by dermatophytes twice as often as domestic short-haired cats. Such cats may have more severe lesions and shed spores for a longer time period [9]. In this study an accelerated recovery from lesions in exotic cats was observed after therapeutic vaccination within the 42 days of the study. The improved healing in these cats showed an increased trend at the last observation point.

A vaccine $\left(\right.$ Insol $^{\circledR}$ Dermatophyton, Boehringer Ingelheim, Germany), similar to the vaccine tested in this study, containing eight fungal strains has been approved in several European countries for the use in horses, cats and dogs for the treatment of dermatophytosis in these species. The vaccine used in this study was based on the same principles, but contained only the strains which are most relevant in cats: T. mentagrophytes, $M$. canis, $M$. canis var. distortum, $M$. canis var. obesum and $M$. gypseum. The total number of microconidia per strain was increased (similarly for each strain) to achieve a higher antigen content of the cat relevant strains, but the total number of microconidia per dose was the same as in Insol ${ }^{\circledR}$ Dermatophyton (Boehringer Ingelheim, Germany) [32, 33].

Further studies with this vaccine, which could include disinfection programs and longer term follow up of fungal cultures, are needed to prove the long term efficacy of this therapeutic vaccine. It would also be interesting to be able to monitor the immune response of the cats to the vaccine, although this is difficult in client owned cats. However, the study results presented here indicate that the therapeutic vaccine is significantly efficacious in accelerating the recovery from lesions of young cats and cats with a first dermatophytosis infection. There was a clear trend of improvement in all severely infected cats following therapeutic vaccination, although this improvement was not significantly different from placebo treated cats $(\mathrm{p}=0.056)$.

\section{CONCLUSION}

The results of this field study with the therapeutic vaccine for cats, representing a variety of breeds and different age ranges have shown that it is efficacious in the treatment of clinical signs of dermatophytosis as measured by the reduction of number and severity of lesions under typical husbandry conditions of privately owned cats. The therapeutic vaccine is able to stimulate healing and to shorten the clinical course of dermatophytosis, which are clear benefits from its use. However, based on this study, infective fungal organisms on the cats are not completely eliminated, but still present as shown by positive fungal cultures. Further studies are needed to establish optimal treatment protocols to achieve reliable microbial remission in infected cats.

\section{ACKNOWLEDGEMENTS}

We would like to thank the investigators, C. Anders, L. Ansay, R. Bauer, P. Cadot, B. Czetoe, M. Heineking, M. Horn, P. Kåber, S. Koebrich, U. Mayer, P. Obschernikat, M. Palm, S. Peters, L. Panakowa, D. Preuss and P. Werhahn for participating in the study. We also like to thank C. Feltrup and S. Kraeft for the laboratory investigations at Serumwerk Memsen, WDT Germany, M. Vanselow for the statistical evaluations and P. Frenchick for review of the manuscript.

\section{CONFLICT OF INTEREST}

During the conduct of the study, D.K. Westhoff, M.-C. Kloes, F.X. Orveillon, D. Farnow, and K. Elbers were either employed or under contract with Boehringer Ingelheim Vetmedica GmbH, Germany. R.S. Mueller has received support and/or funding for research studies and consulting activities from Bayer Animal Health, Boehringer Ingelheim Animal Health, DVM Pharmaceuticals, Laboratoire de Dermo-Cosmétique Animale, Novartis Animal Health, Pfizer Animal Health, Proctor and Gamble Pet Care, Royal Canin and Virbac. The study and all related work was funded by Boehringer Ingelheim Vetmedica $\mathrm{GmbH}$, Germany.

\section{REFERENCES}

[1] Ben-Ziony Y, Arzi B. Use of lufenuron for the treating of fungal infections of dogs and cats: 297 cases (1997-1999). J Am Vet Med Assoc 2000; 217: 1510-3.

[2] Mancianti F, Nardoni S, Cecchi S, Corazza M, Taccini F Dermatophytes isolated from symptomatic dogs and cats in Tuscany, Italy during a 15-year-period. Mycopathologica 2002; 156: $13-8$

[3] Cafarchia C, Romito D, Sasanelli M, Lia R, Capelli G, Otranto D. The Epidemiology of canine and feline dermatophytosis in southern Italy. Mycoses 2004; 47: 508-13.

[4] Moriello KA. Treatment of dermatophytosis in dogs and cats: review of published studies. Vet Dermatol 2004; 15: 99-07.

[5] Chermette R, Ferreiro L, Guillot, J. Dermatophytoses in animals. Mycopathologica 2008; 166; 385-05.

[6] Cabanes FJ, Abarca ML, Bragulat MR. Dermatophytes isolated from domestic animals in Barcelona, Spain. Mycopathologica 1997; 137: 107-13.

[7] Brilhante RSN, Cavalcante CSP, Soares-Junior FA, Cordeiro RA, Sidrim JJC, Rocha MFG. High rate of Microsporum canis feline and canine dermatophytosis in Northeast Brazil: epidemiological and diagnostic features. Mycopathologica 2003; 156: 303-8.

[8] Lewis DT, Foil CS, Hosgood G. Epidemiology and clinical features of dermatophytosis in dogs and cats at Louisiana State University: 1981-1990. Vet Dermatol 1991; 2: 53-8.

[9] DeBoer DJ, Moriello KA. Clinical update on feline dermatophytosis - Part I. J Small Anim Pract 1995; 17: 1197-03.

[10] Sparkes AH, Gruffydd-Jones TJ, Shaw SE, Wright AI, Stokes CR. Epidemiological and diagnostic features of canine and feline dermatophytosis in the United Kingdom from 1956 to 1991. Vet Rec 1993; 133: 57-61.

[11] Mancianti F, Giannnelli C, Bendinelli M, Poli A. Mycological findings in feline immunodeficiency virus-infected cats. J Med Vet Mycol 1992; 30: 257-9.

[12] Mancianti F, Nardoni S, Corazza M, Achille PD, Ponticelli C. Environmental detection of Micropsorum canis arthrospores in the housholds of infected cats and dogs. J Feline Med Surg 2003; 5: 323-8.

[13] DeBoer DJ, Moriello KA, Cairns R. Clinical update on feline dermatophytosis - Part II. J Small Anim Pract 1995; 17: 1471-80.

[14] Kotnik T. Drug efficacy of terbinafine hydrochloride (Lamisil@) during oral treatment of cats, experimentally infected with Microsporum canis. J Vet Med 2002; B49: 120-2.

[15] Mancianti F, Pedonese F, Zullino C. Efficafy of oral administration of itraconazole to cats with dermatophytosis caused by Micropsorum canis. J Am Vet Med Assoc 1998; 213: 993-5.

[16] Guillot J, Malandain E, Jankowski F, et al. Evaulation of the efficacy of oral lufenuron combined with topical enilconazole for the management of dermatophytosis in catteries. Vet Rec 2002; 150: 714-8. 
[17] Conant NF, Martin DS, Smith DT, Baker RD, Callaway JL. Manual of clinical mycology. Philadelphia \& London: WB Saunders Co. 1944

[18] Beemer AM, Kuttin ES, Pinto M. Treatment with antifungal vaccines. Control Microbiol Immunol 1977; 4: 136-46.

[19] Deepe GS. Prospects for the development of fungal vaccines. Clin Microbiol Rev 1997; 10: 585-96.

[20] Mignon B, Tabart J, Baldo A, Mathy A, Losson B, Vermout S. Immunization and dermatophytosis. Curr Opin Infect Dis 2008; 21: 134-40.

[21] Lund A, DeBoer DJ. Immunoprophylaxis of Dermatophytosis in animals. Mycopathologica 2008; 166: 407-24.

[22] Gudding R, Naess B. Vaccination of cattle against ringworm caused by Trichophyton verrucosum. Am J Vet Res 1986; 47: 2415-7.

[23] Vermout SM, Brouta FD, Deschamps FF, Losson BJ, Mignon BR. Evaluation of immunogenicity and protective efficacy of a Microsporum canis metalloprotease subunit vaccine in Guniea pigs. FEMS Immunol Med Microbiol 2004; 40: 75-80.

[24] Fenner A, Karle J. Therapeutic vaccination with insol® dermatophyton against dermatophytosis in horses. Der praktische Tierarzt 2000; 81: 574-9.

[25] Rybnikar A, Vrzal V, Chumela J. Protective efficacy of vaccines against bovine dermatophytosis after double and single vaccination. Mycoses 1998; 41: 83-6.

[26] Bredahl LK, Panin AN, Solbakk IT, Lund A. Safety of an experimental Microsporum canis vaccine in farmed foxes. Vet Dermatol 2000; (11 Suppl 1): 45.

[27] DeBoer DJ, Moriello KA. The immune response to Microsporum canis induced by a fungal cell wall vaccine. Vet Dermatol 1994; 5: 47-55.
[28] DeBoer DJ, Moriello KA. Investigations of a killed dermatophyte cell-wall vaccine against infection with Microsporum canis in cats. Res Vet Sci 1995; 59: 110-3.

[29] Pier AC, Hodges AB, Lauze JM, Raisbeck M. Experimental immunity to Micropsorum canis and cross reactions with other dermatophytes of veterinary importance. J Med Vet Mycol 1995; 33: 93-7.

[30] Manoyan MG, Panin AN, Letyagin KP. Effectiveness of microderm vaccine against dermatophytes in animals. Vet Dermatol 2000; (11 Suppl 1): 59.

[31] DeBoer DJ, Moriello AK, Blum JL, Volk LM, Bredahl LK. Safety and immunologic effects after inoculation of inactivated and combined live-inactivate dermatophytosis vaccines in cats. Am J Vet Res 2002; 63: 1532-7.

[32] Boehringer Ingelheim Vetmedica GmbH. Product information Insol $^{\circledR}$ Dermatophyton [cited 2010 Jan 10] Available from: http://www.boehringer-ingelheim.com/products/animal_health. html

[33] Paul Ehrlich Institut Langen, Germany. Licensed vaccines for animals in Germany [cited 2010 Jan 10] Available from: http://www.pei.de/cln_092/nn_161794/DE/arzneimittel/vet$\mathrm{mittel} / \mathrm{katzen} / \mathrm{katzen}-\mathrm{node} . \mathrm{html}$ ?__nnn=true

[34] Scott DW, Horn RT. Zoonotic dermatoses of dogs and cats. Vet Clin North Am Small Anim Pract 1987; 17: 117-44.

[35] Chappuis G. Neonatal immunity and immunisation in early age: lessons from veterinary medicine. Vaccine 1998; 16: 1468-72.

[36] Day MJ. Immune system development in the dog and cat. J Comp Pathol 2007; 137: 10-5.

[37] Schulz RD. Current and future canine and feline vaccination programs. Vet Med 1998; 93: 233-54.

(c) Westhoff et al.; Licensee Bentham Open.

This is an open access article licensed under the terms of the Creative Commons Attribution Non-Commercial License (http://creativecommons.org/licenses/bync/3.0/), which permits unrestricted, non-commercial use, distribution and reproduction in any medium, provided the work is properly cited. 